\title{
Bacterial Enterotoxins
}

\section{Bidjeh Kebkiba*}

Livestock Research Institute for Development (LRID), Ministry of Livestock and Animal Productions, N'Djamena, Chad

*Corresponding Author: Bidjeh Kebkiba, Livestock Research Institute for Development (LRID), Ministry of Livestock and Animal Productions, N'Djamena, Chad.
Received: March 22, 2021

Published: April 06, 2021

(C) All rights are reserved by Bidjeh Kebkiba.

\begin{abstract}
Bacterial enterotoxins are proteins released in food before ingestion. They are produced by pathogenic bacteria, such as Staphylococcus aureus, Echerichia coli, Vibrio Cholerae, Salmonella sp, Shigella dysenteriae, Yersinia enterolytica, and more rarely Clostridium difficile or Clostridium perfringens. These proteins are toxic substances, which act on the intestines of humans or animals. They are therefore harmful to human and animal health. These proteins are most often responsible for food poisoning. They differ significantly from each other in terms of both their structure and their mechanism of action. Some of them work by activating a cycladic system in the enterocyte, causing water and electrolytes to flow into the intestinal lumen, resulting in dehydration, decreased weight gain, and/or death in animal or human. Intestinal infections and the diarrhea associated with enterotoxins represent in developing countries in particular, a major cause of infant mortality. Foods wich facilitate the growth of bacteria are mainly cream pastries, ice cream, processed foods such as hams, pasta and rillettes; potato, poultry and tuna salads, raw vegetables. This article describes the germs producing enterotoxins, the environments favorable to the development of the germs producing these proteins, types of infections caused by bacterial enterotoxins, different types of enterotoxin-producing bacteria, different types of bacterial enterotoxins, clinical signs observed in humans and diagnosis.
\end{abstract}

Keywords: Enterotoxins; Staphylococcus aureus; Clostridia; Salmonella; Vibrio cholerae; Echerichia coli

\section{Abbreviations}

LT: Heat-labile; ST: Heat-stable; LPS: Lipopolysaccharides; ECET: Enterotoxinogenic E. coli; ECEI: Entero-invasive E. coli; cAMP: Cyclic Adenosine Monophosphate; ELISA: Enzyme-Linked Immunosorbent Assay; Ng: Nanogram

\section{Introduction}

Defined as toxic substances, Enterotoxins are macroproteins produced by pathogenic bacteria that act on the intestines of humans or animals. They are most often responsible for diarrhea (whether or not associated with tissue damage). These molecules differ significantly from each other both in their structure and in their mechanism of action. Some of them, such as cholera toxin, the heat-labile (LT) and heat-stable (ST) enterotoxin of Escherichia coli, act by activating a cycladic system (adenylate or guanylate cyclase) in the enterocyte and cause a flow of water and electrolytes in the enterocyte in the intestinal lumen, resulting in dehydration, decreased weight gain, and/or death in animal or human [1]. On the other hand, Clostridium perfringens enterotoxin, toxins A and $\mathrm{B}$ of Clostridium difficile, verotoxin of shigella dysenteriae cause disruption of certain cellular functions such as protein synthesis, permeability to small molecules; they also cause tissue damage in the 
gut [1]. Intestinal infections and the diarrhea associated with them represent, in developing countries in particular, a major cause of infant mortality. It is currently estimated that nearly 5 million children under the age of 5 years die each year from these conditions [1]. In addition, serious digestive tract infections have also been noted in pigs and calves in particular [2]. According to Burgess., et al. [3]; Greenburg and Guerrant [4], these epidemics are attributed, for the most part to enterotoxigenic strains of Escherichia Coli, which produce heat-labile (LT) and heat-stable (ST) enterotoxins. Note that most of these enterotoxins act through a membrane receptor which they specifically recognize, these molecules act on the wall of the intestine, hence the name enterotoxin used to define them [1]. They are low molecular weight proteins. The objective of this article is to summarize the information generated from the bibliographic data.

Media favorable to the development of bacteria producing enterotoxins

Foods, which facilitate the growth of bacteria are mainly cream pastries, ice cream, processed foods such as hams, pasta and rillettes; potato, poultry and tuna salads, raw vegetables [22]. Cooked products contaminated after cooking (minced meat, fish, cold cuts); products with reduced water content (e.g. cured meats, dried and smoked fish, powdered milk), cheeses, following insufficient acidification of the curdled milk, egg products, mayonnaise, dairy products (e.g. condensed milk, creams, ice creams, etc.). Contaminated food looks the same (looks, smells, tastes) as healthy food [23]. There have even been poisonings after consuming canned vegetables and fruits [24].

\section{Diversity of enterotoxins}

Enterotoxins act primarily in the gut. Enterotoxins exhibit great diversity in structure, molecular weight and mechanism of action. Bacterial enterotoxins are generally classified according to their biochemical and structural characteristics on the one hand, molecular mass, number of subunits, sensitivity to heat or enzymes and, on the other hand, according to their mechanism of action or their types of biological activities [1,25]. Based on the latter classification, these molecules can be distinguished into two groups: cytotonic enterotoxins and cytotoxic enterotoxins [25]. Cytotonic enterotoxins affect intestinal epithelial cells to directly alter their secretory functions $[5,6]$. The main character of these enterotoxins lies in their power to induce a secretory response in ligated intes- tinal loops in an animal model $[7,8]$. The interaction of enterotoxins with the intestinal mucosa leads either to a direct effect on the cell wall of the host cell or to a disruption of the cellular signaling pathways. However, before even affecting the target cell, the toxin secreted by the micro-organism will have to recognize and attach to a molecule present on the cell surface, its receptor [1]. The membrane receptors for bacterial enterotoxins can be protein, glycoprotein or glycolipid in nature. The different enterotoxins liable to cause intestinal disorders during its diffusion in the digestive system are: Salmonella enterotoxin, staphylococcal enterotoxins, vibrio cholerae enterotoxin, Shigella dysenteriae enterotoxin, Escherichia Coli enterotoxins, Clostridium perfringens enterotoxin, Bacillus cereus enterotoxins, etc.

Presentation of enterotoxin-producing micro-organisms Salmonella

Salmonella are the bacteria most frequently involved in food poisoning. The infectious dose must be greater than the defense capacities of the digestive tract, and it is assumed that the minimum infective dose is generally higher or equal to $10^{5}$ bacteries. The most frequently implicated foods are eggs (S. enteritidis), meat, more particularly poultry, and dairy products [9]. The contaminating food should be eaten raw or undercooked. Clinically salmonellosis is manifested by febrile diarrhea accompanied by vomiting and abdominal pain and moderate fevers [1]. They can cause bacteraemia and are complicated by septicemia or secondary extradigestive localizations which severity of illness. These symptoms usually appear after an incubation period of 24 - 48 hours [15].

\section{Staphylococcus aureus}

Staphylococcus aureus is a commonly recognized cause of food poisoning. Their reservoir is usually human. Food contamination by this germ occurs during the preparation by a healthy carrier (nasopharyngeal carriage) or with a wound infected with Staphylococcus aureus; phage group III and IV (boils, panaris). Heat-stable enterotoxin is produced within the food and it is only this toxin and not the Staphylococcus, which is responsible for the disorders. Staphylococcal infections are disease, which is more frequently associated with dairy products such as cheese, milk, ice cream $[26,27]$ or dishes (salads compound, dried meats) that have undergone significant handling [9]. Staphylococcus is a halophilic germ (possible growth in a salty environment). 
The pathogenicity of Staphylococcus aureus is also linked to the production of toxins called enterotoxins. These, named by letters from $A$ to $E$ (staphylococcal enterotoxins A, B, C, D, E, F...), are preformed in the food during bacterial multiplication [28]. These different enterotoxins have been demonstrated. To date, only six (6) types of staphylococcal enterotoxins $(\mathrm{A}-\mathrm{H})$ have been identified. Staphylococcal enterotoxins are resistant to $100^{\circ} \mathrm{C}$ for 30 minutes and gastric acidity [9]. These different serotypes are antigenetically distinct. However, some authors such as Bergdoll., et al. $[8,10]$, have shown that there is an antigenic relationship between staphylococcal enterotoxins type A and E; enterotoxins type B and C [11]. These authors believe that the molecules of these enterotoxins contain fractions of antigens (sites) determining only the specificity of the groups. Thus, type A and E; B and C enterotoxins are antigenically linked to each other.

\section{Shigella dysenteriae}

Shigella dysenteriae type 1 is involved in humans in certain diarrhea of a sometimes dysenteric nature, due to the entero-invasive nature of this bacterium. It produces a major enterotoxin in the intestine known as shigatoxin or verotoxin, due to the toxic effect that this molecule has on Vero cells. This $70 \mathrm{kDa}$ toxin consists of 2 components. The A subunit is a $30.5 \mathrm{kDa}$ polypeptide carrying the toxic activity of the protein cleavable into $2 A_{1}$ and $A_{2}$ subunits of 27.5 and $3 \mathrm{kDa}$, respectively. The second component of this toxin consists of 4-5 B subunits, of 7-11 kDa each [12-14]. This structure is analogous to those of cholera toxin or of E. coli LT, although the verotoxin does not show antigenic relatedness to the latter.

\section{Vibrio cholerae}

Cholera is an infectious diarrheal disease of epidemic nature, originating bacterial, transmitted through the digestive tract. It is the disease of faecal peril, real emergency in public health. Cholera is the privileged companion of natural disasters and conflict situations with massive displacement of populations. However, it can occur in a context of political stability and in the absence of any natural calamity, when the socio-economic conditions of the populations are favorable to its development.

Vibrio cholerae, a germ that develops in the small intestine in humans produces cholera toxin, during intestinal infections caused by it. Cholera toxin is responsible for very severe diarrhea. This once dreaded disease is now fatal only in underserved areas of the world.

\section{Escherichia coli}

The pathogenic E. coli found in each pathotype can be grouped into different virotypes, depending on the virulence of genes they have. A virotype is a specific combination of virulence of genes. Among the most important virulence factors encoded by these genes are the fimbrial adhesins, enterotoxins, cytokines, capsule and lipopolysaccharides (LPS). Different pathogenic E. coli can also be distinguished by serotyping based on antigenic differences in LPS $\mathrm{O}$ antigen, flagellum $\mathrm{H}$ antigen, and fimbriae $\mathrm{F}$ antigen.

E. coli is responsible for sometimes hemorrhagic intestinal infections in humans and animals, mainly in pigs. We have also characterized many pathogenic strains of this bacterium, enterotoxinogenic (ECET) or entero-invasive (ECEI, 0157: H7) [15]. The toxigenic strains produce several types of toxins, including: a heatlabile enterotoxin (LT) and heat-stable toxins (STa and STb), whose mechanisms of action are similar but have totally different structures [16].

\section{Clostridium perfringens}

Clostridium perfringens is frequently involved in catering when the rules for storing food after cooking have not been observed. Half of approximately case is due to mixed foods, most often meats in sauce or dishes compound, $95 \%$ of cases are related to cooked products. They are heat-resistant spore-forming bacteria that germinate and multiply when there are favorable conditions (sufficiently long time between preparation and consumption of food, temperature and anaerobiosis). Meats in sauce are therefore frequent means of contamination.

There are 5 classes of $C$. perfringens (A-E). This classification is based on the ability of these bacteria to produce different toxins or toxic factors. C. perfringens is responsible for toxic infections in humans, characterized by diarrhea and abdominal cramps that begin 10-12 hours after ingestion of contaminated food. Enterotoxin produced by A, D or C strains is associated with Clostridium sporulation. This molecule consists of a single polypeptide chain of 34 $\mathrm{kDa}$ [17]. It is thermolabile, its toxic power is notably destroyed by heating.

\section{Clostridium difficile}

During infection, in humans or animals, $C$. difficile usually produces 2 toxins. Enterotoxin or toxin A, which is assayed using an ELISA test [18]. Toxin A exerts a cytotonic action in the ligated 
intestinal loop [19] but also cytotoxic to a lesser extent, when its activity is evaluated on cell cultures $[20,21]$. The cytotoxin or toxin $\mathrm{B}$ is approximately 1000 times more toxic than toxin A to certain cell lines.

\section{Bacillus cereus}

Bacillus cereus can cause food poisoning. Rice, pasta, meat, poultry, vegetable dishes, various soups and sauces can cause such poisoning. This germ can cause two types of conditions: "emetic syndrome" mainly with cooked rice, This syndrome is caused by diarrheal enterotoxin.

\section{Diversity of enterotoxin-producing bacteria}

The list of pathogenic microorganisms producing enterotoxins is long, we will limit ourselves here to mentioning the most common: Salmonella, Staphylococcus, Shigella dysenteriae, vibrio cholerae, Escherichia Coli, clostridii, Yersinia enterocolitica, bacillus cereus, Campylobacter, etc. These bacteria are well known microorganisms. Some of them produce toxins causing serious illnesses such as tetanus, cholera, diphtheria, typhoid fever, etc. They produce two types of toxins called endotoxins and exotoxins. Enterotoxins are therefore exotoxins that are released in the intestines of organisms; they are produced and released outside of bacterial cells. Enterotoxins are produced by certain bacterial species and cause food poisoning and several bowel diseases. In general, enterotoxins are produced by Gram-positive bacteria. However, some Gram-negative bacteria such as Escirichia coli, Vibrio cholerae can also produce enterotoxin.

\section{Origin of bacteria causing food poisoning}

Failure to respect the cold chain, errors in the preparation process of food and too long time between preparation and consumption are the main factors favoring the occurrence of food poisoning. Diseases caused by enterotoxins (enterotoxicosis) are of bacterial origin, acute infectious diarrhea are transmissible diseases, which almost always participate in a faecal-oral cycle. The epidemiological modalities vary from one continent to another depending on the level of development of health infrastructures. In countries with poor hygiene, bacterial diarrhea is responsible for more than four (4) million deaths per year. Their transmission is favored by the absence of collective equipment necessary for the elimination of wastewater and the distribution of drinking water, but the exchange of enteric germs results mainly from direct human-to-hu- man contact and frequent pollution of the environment and drinking water. In industrialized countries where the sanitary equipment is satisfactory, the direct transmission of enteric germs is rare and their dissemination through drinking water is exceptional. On the other hand, we are witnessing an increase in food poisoning, reflecting major sociological changes that have occurred over the past quarter of a century. The emergence of collective catering and new methods of production, processing and distribution of food has deeply changed individual and collective behavior regarding meal times and places. These changes, accompanied by new requirements with regard to the quality of products, the hygiene of their environment and consumer safety, are at the origin of the development of food bacteriology. The foods involved are very varied. These are often cold meats, drinking water, eggs, fruit and dairy products [23,26,27]. Contamination, almost always exogenous and of human origin, consists of a lack of hygiene, which can be located at any point in the food chain. The main germs concerned are multiple. These bacteria are often from food or waterborne and cause infectious food poisoning. The risks of transmitting these bacteria are linked to the consumption of water and food. Pathogenic bacteria that contaminate the environment are ingested by susceptible animals or humans and enter the intestinal tract. These bacteria have fimbriar adhesins that allow adhesion to specific receptors on epithelial cells in the intestine. This bacterial colonization is found mainly on the mucous membranes of the jejunum and/or the ileum. The adhered bacteria produce which cause loss of water and electrolyte in the intestinal lumen, resulting in dehydration, decreased weight gain, and/or death in animal or human.

\section{Enterotoxicosis (diseases caused by enterotoxins)}

Of bacterial origin in 60 to $80 \%$ of cases, acute infectious diarrhea are transmissible diseases, which almost always participate in a faecal-oral cycle. The epidemiological modalities vary from one continent to another depending on the level of development of health infrastructures.

\section{Clinical manifestations}

Incubation period is brief one to four hours ( 1 - 4h). Symptoms appear suddenly, sometimes even before the end of the contaminating meal. They create a picture of acute gastroenteritis with nausea, vomiting, abdominal pain, diarrhea and some times Neurological disorders. There is no fever, but the risk of dehydration is high. Arterial hypotension, cardiovascular collapse can complicate 
the course. These rare accidents occur at the extreme ages of life. Typically, symptoms improve within six to eight hours (6 - 8h).

Staphylococcal enterotoxins increase the intracellular concentration of cyclic adenosine monophosphate (cAMP). This results in a disruption of cellular electrolyte exchanges causing diarrhea, but no cellular damage or villous destruction is observed. Diarrhea results from the ingestion of a preformed bacterial toxin in the food. The onset of symptoms is early after absorption of the food. There is a dose-effect relationship, the damage being all the more severe and the incubation period shorter than the quantity of toxins ingested would have been important.

The amount of enterotoxin ingested (in the digestive tract) determines when symptoms appear, as well as their severity. It takes 500,000 to 5, 000,000 germs/g to trigger the troubles. Symptoms appear quickly ( 1 to $2 \mathrm{~h}$, up to $6 \mathrm{~h}$ ); at the start, it is profuse salivation, nausea, headache, vomiting (violent, incoercible and repeated), sweat, neurological disorders (prostration to collapse), severe abdominal pain and severe diarrhea. There is generally no sharp rise in temperature, sometimes a slight hyperthermia (up to $38^{\circ}$ C), or on the contrary hypothermia (severe cases in infants and the elderly). Severe cases are accompanied by dehydration and discharge of blood and mucus in the stool and vomiting. Experience in humans weighing $70 \mathrm{~kg}$ has shown that symptoms appear from 3.5 ng of enterotoxins consumed [28]. However, it is estimated that in sensitive subjects $100 \mathrm{ng}$ may be sufficient to trigger clinical signs. Animals are less sensitive than humans to the action of enterotoxins.

\section{Diagnosis}

In the food industry, professionals ensure compliance with regulatory criteria to ensure consumer safety. But since enterotoxins are resistant to most treatments used, they remain in food despite freezing or cooking. It is therefore important to detect them. Until now, detection of staphylococcal enterotoxins and other bacterial enterotoxins have been done by biological and immunochemical tests [29]. The immunochemical detection method is carried out after extraction and then dialysis. These different methods used have posed communication and trade problems between countries when controlling food at borders. To meet the need for harmonization and international regulatory requirements, a group of experts was set up to develop a validated enterotoxin detection method for five food categories. With financial support from the European Union, the first standardized enzyme-linked immunosorbent assay (ELISA) method has been developed. These kits are marketed. This first standardized detection method is now at the heart of the draft European and international voluntary standard in ISO 19020 defining performance criteria for diagnostic kits [28]. With this voluntary European standard, laboratories will be able to analyze food products. The immuno-enzymatic method proposed consists in extracting and then concentrating the enterotoxins in order to better identify them by the said test.

\section{Conclusion}

Salmonella, Staphylococci, Shigella dysenteriae, Vibrio cholerae, Escherichia Coli, Clostridii, Yersinia enterocolitica, Bacillus cereus and Campylobacter are pathogens, which cause food poisoning around the world. The major vectors of these germs are products made from milk, meats, eggs, water and vegetables. For example, carriage by cows suffering from mastitis is the source of contamination of milk and its products by staphylococci and $E$. coli. Heat treatment, respect for good hygiene practices and respect for the cold chain are of course the major axes allowing the considerable reduction of bacterial germs in food, but however have no effect on enterotoxins.

\section{Conflict of Interest}

By this I declare that there is no conflict of interest.

\section{Bibliography}

1. Lucas F., et al. "Bacterial enterotoxins: structure and mechanism of action". Annal of Veterinary Research 22.2 (1991): 147162.

2. Smith JA., et al. "Clostridium difficile toxin A binding to human intestinal epithelial cells". Journal of Medical Microbiology 5.46 (1997): 953-958.

3. Burgess MN., et al. "Biological evaluation of a methanol soluble, heat-stable E coli enterotoxin in infant mice, pigs, rabbits, and calves". Infection and Immunity 21 (1978): 526-553.

4. Greenberg RN., et al. "Comparison of effects of uroguanylin and Escherichia coli heat-stable enterotoxin in mouse intestine and kidney: evidence that uroguanylin is an intestinal natriuretic hormone". Journal of Investigative Medicine 45 (1997): 276282. 
5. Gemmell CG. "Comparative study of the nature and biological activities of bacterial enterotoxins". Journal of Medical Microbiology 17 (1984): 217-235.

6. Fasano A., et al. "Enterotoxin and cytotoxin production by entero-invasive Escherichia coli". Infection and Immunity 58 (1990): 3717-3723.

7. Bergdoll MS. "Ileal loop fluid accumulation test for diarrheal toxins". Methods Enzymology 165 (1988): 306-323.

8. Bergdoll MS and Robbins RN. "Characterization of staphylococcal entérotoxin types". Journal of milk and Food Technology 36 (1973): 610-612.

9. Bidjeh K and Podkapaev VM. "Isolation and Identification of Staphylococcal strains Producing Enterotoxin D in Cow Milk and Feaces of Calves Suffering from Enteritis". International Journal of Current Microbiology and Applied Science 49 (2015): 528-534.

10. Lee ACM and Robbins RN. "Isolation of specific and common for staphylococcal enterotoxin A and $\mathrm{E}$ by affinity chromatography". Infection and Immunity 21.2 (1978): 387-391.

11. Gruber J and Wright CC. "Ammonium sulfate coprecipitation antibody determination with purified staphylococcal enterotoxins". Journal of Bacteriology 99 (1969): 18-24.

12. Olsnes S., et al. "Subunit structure of Shigella cytotoxin". Journal of Biological Chemistry 256 (1981): 8732-8738.

13. Keusch GT., et al. "Shiga toxin: production and purification". Methods enzymology 165 (1988): 152-162.

14. Ryd M., et al. "Purification of Shigatoxin by -D-galactose- (1-4)p-D-galactose- (1-4)- (3-D-glucose- (1-) receptor ligand-based chromatography". FEBS Letter 258 (1989): 320-322.

15. Riley LW. "The epidemiologic, clinical, and microbiologic features of hemorrhagic colitis". Annual Review of Microbiology 4.1 (1987): 383-407.

16. Giannella RA., et al. "Binding of Escherichia coli heat-stable enterotoxin to receptors on rat intestinal cells". American Journal of Physiology 2.45 (1985): 492-498.
17. Hanna PC and McClane BA. "A recombinant C-terminal toxin fragment provides evidence that membrane insertion is important for Clostridium perfringens enterotoxin cytotoxicity". Molecular Microbiology (1991): 225-230.

18. Lyerly DM., et al. "Enzyme-linked immunosorbent assay for Clostridium difficile toxin A". American Journal of Clinical Microbiology 17 (1983): 72-78.

19. Triadafilopoulos G., et al. "Comparative study of Clostridium difficile toxin A and Cholera toxin in rabbit ileum". Gastroenterology 97 (1989): 1186-1192.

20. Libby JM., et al. "Effects of the two toxins of Clostridium difficile in antibiotic-associated cecitis in hamsters". Infection and Immunity 36 (1982): 822-829.

21. Corthier G., et al. "Interrelationships between digestive proteolytic activities and production and quantitation of toxins in pseudomembranous colitis induced by Clostridium difficile in gnotobiotic mice". Infection and Immunity 57 (1989): 39223927.

22. Seo KS and Bohach GA. "Staphylococcus aureus". In: Food microbiology: Fundamentals and Frontiers, Doyle, M.P. and L.R. Beuchat eds. ASM press, W. D.C, USA (2007): 493-518.

23. Debuyser ML., et al. "Involvement of milk and milk products in food borne diseases in France and different industrialized countries". International Journal of Food Microbiology 67 (2001): 1-17.

24. Afssa. published hazard sheets by Afssa, ed. Afssa, Maisons Alfort France (2009): 106.

25. Eidels L., et al. "Membrane receptors for bacterial toxins". Microbiology Review 47 (1983): 596-620.

26. Debuyser ML., et al. "Involvement of milk and milk products in food borne diseases in France and different industrialized countries". International Journal of Food Microbiology 67 (2001): 1-17.

27. Merzaei HH., et al. "Presence and antimicrobial susceptibility of methicillin-resistant Staphylococci in raw milk and ice cream in Tabriz by culture and PCR techniques". African Journal of Microbiology Research 6 (2012): 6224-6229. 
28. Bidjeh K., et al. "Staphylococcal enterotoxins". Journal of Microbiology Research 8.1 (2018): 19-22.

29. Bidjeh K. "Development of method for identification of staphylococcal enterotoxin type D in milk/colostrum of cows and in the feaces of newborn calves". PhD thesis in veterinary science (microbiology), Moscow (1984): 176.

\section{Assets from publication with us}

- Prompt Acknowledgement after receiving the article

- Thorough Double blinded peer review

- Rapid Publication

- Issue of Publication Certificate

- High visibility of your Published work

Website: www.actascientific.com/

Submit Article: www.actascientific.com/submission.php

Email us: editor@actascientific.com

Contact us: +919182824667 